\title{
Traducción, adaptación y validación de un instrumento de autoevaluación para alumnado universitario: hacia el éxito en el aprendizaje
}

\section{Elena Escolano-Pérez ${ }^{\mathrm{a}}$, Rita Pilar Romero Galisteo ${ }^{\mathrm{b}}$ y Pablo Gálvez Ruiz}

${ }^{a}$ Universidad de Zaragoza. Facultad de Educación. Departamento de Psicología y Sociología, eescola@unizar.es, bUniversidad de Málaga. Facultad de Ciencias de la Salud. Departamento de Fisioterapia, rpromero@uma.es, 'Universidad Internacional de La Rioja. Facultad de Educación. Departamento de Educación en Internet, pablo.galvez@unir.net.

\begin{abstract}
Many university students have difficulties in their autonomous learning due to deficits in their executive functions, which leads to a negative impact on their training and academic performance. Thus, early detection of students with such difficulties will allow the design of actions for improvement, contributing ultimately to a greater academic and work success. However, in the Spanish university context, there is a lack of reliable and valid instruments to carry out an evaluation of the executive functions, so the objective of this study is to translate, adapt and validate to the university population of new entry in the University of Zaragoza, an instrument for the assessment of the executive functions (Amsterdam Executive Function Inventory). The results showed adequate psychometric properties, with a good internal consistency and a goodness of fit adjustment in the indices considered of the confirmatory factor analysis. Thus, the instrument used is a reliable and valid to identify students with difficulties in executive functions and therefore, students are likely to have problems in their autonomous learning, which will allow not only the identification of the same, but the ultimate aim of being able to intervene for their improvement and optimization.
\end{abstract}

Keywords: Autonomous Learning, Executive Functions, European Higher Education Area, beginner university students.

\section{Resumen}

Muchos estudiantes universitarios presentan dificultades en su aprendizaje autónomo debidas a su vez a déficits en sus funciones ejecutivas, lo que conlleva repercusiones negativas en su formación y rendimiento académico. Así, detectar tempranamente estudiantes con dichas dificultades permitirá diseñar acciones para su mejora, contribuyendo en última instancia a un mayor éxito académico y laboral. Sin embargo, en el contexto universitario español se carece de instrumentos fiables y válidos que permitan llevar a cabo una evaluación de las funciones ejecutivas, por lo que el objetivo de este estudio es traducir, adaptar y validar para la población universitaria de 
nuevo ingreso en la Universidad de Zaragoza un instrumento de evaluación de las funciones ejecutivas (Amsterdam Executive Function Inventory). Los resultados mostraron adecuadas propiedades psicométricas, con una buena consistencia interna y un buen ajuste en los índices considerados del análisis factorial confirmatorio. Así, la herramienta empleada supone un instrumento fiable y válido a la hora de identificar alumnado con dificultades en las funciones ejecutivas y por tanto, alumnado con probabilidad de presentar problemas en su aprendizaje autónomo, lo que permitirá no sólo la identificación de los mismos, sino la finalidad última de poder intervenir para su mejora y optimización.

Palabras clave: Aprendizaje autónomo, funciones ejecutivas, Espacio Europeo de Educación Superior, alumnado universitario de nuevo ingreso.

\section{Introducción}

El aprendizaje autónomo constituye un concepto clave en la Educación Superior, entendiéndose como tal aquel aprendizaje en el que el estudiante se responsabiliza de la organización de su trabajo y de la adquisición de las diferentes competencias según su propio ritmo. Implica, por tanto, que quien aprende debe asumir la responsabilidad y el control de las decisiones sobre la planificación, realización y evaluación de sus actividades (Francis y Flanigan, 2012). Es preciso remarcar que la autonomía en el aprendizaje se concede con respecto a las condiciones pero no sobre su resultado o producto. El estudiante debe dar respuesta a las demandas específicas de conocimiento formuladas por el docente, pero escogerá por sí mismo aquellas condiciones contextuales de tiempo, lugar, herramientas, etc. que estime necesarios para elaborar aquella respuesta (Rué, 2009). El docente se convierte en guía, compañero y apoyo en ese proceso de aprendizaje del alumno. Su tarea esencial consiste en diseñar tareas, situaciones y contextos de aprendizaje lo más cercanas posible al contexto real que supongan un desafío a los estudiantes, orientando y ayudando a estos en la construcción de unas estructuras mentales que les permitan gestionar la información disponible de manera óptima, integrando y relacionando las demandas externas, el contexto y las características personales en una estructura de significado que dé respuesta al problema concreto al que se enfrenta en cada caso. Estos problemas, así como la información disponible para su solución, van a ser continuamente cambiantes, pues así lo es el contexto en el que vivimos. Nuestra sociedad se caracteriza por su cambio constante (Pozo y Monereo, 2009), lo cual imposibilita que el estudiante, durante su etapa universitaria o incluso durante toda su educación reglada, pueda adquirir todos los conocimientos que necesitará en su futura labor profesional. Ni siquiera resulta posible predecir cuáles serán estos conocimientos específicos. Por tanto, la formación universitaria no puede dotar a sus estudiantes de todos los conocimientos y saberes imprescindibles en su futuro profesional. Sin embargo, lo que puede y debe hacer es formar a los futuros profesionales para que sean aprendices más flexibles, eficaces y autónomos, dotándoles para ello de capacidades de aprendizaje y no sólo de conocimientos o saberes específicos, muchos de ellos, probablemente, obsoletos en un futuro no demasiado lejano (Pozo y

(cc) EY-NC-ND 2018, UniversitatPolitècnica de València

Congreso IN-RED (2018) 
Monereo, 2009). El alumno, más que centrarse con exclusividad en la adquisición y dominio de conocimientos y saberes, debe hacerlo en el desarrollo de habilidades y procesos cognitivos que le ayuden a resolver de manera creativa y eficaz los problemas y exigencias que se le presenten en esta sociedad del cambio en la que vivimos. Es en este sentido que distintos autores defienden que el aprendizaje autónomo de los alumnos se encuentra estrechamente unido a sus funciones ejecutivas (Zelazo, Blair y Willoughby, 2016), entendidas estas como procesos cognitivos de alto nivel que permiten la coordinación de sus pensamientos, emociones y actos durante la resolución activa de un problema, especialmente en aquellos problemas que resultan complejos y requieren un enfoque novedoso (Carlson, Zelazo y Faja, 2013; Diamond, 2013; Miyake y Friedman, 2012).

Así, las "funciones ejecutivas" nos permiten organizar y planificar una tarea, seleccionar apropiadamente los objetivos, iniciar un plan y mantenerlo en la mente mientras lo ejecutamos, controlarnos para no responder automáticamente, inhibir o resistir las distracciones, detectar los errores, cambiar de estrategias de modo flexible, considerar las consecuencias de las acciones, autorregular y controlar el curso de nuestra acción para asegurarnos de que la meta propuesta está en vías de conseguirse, reflexionar sobre las experiencias pasadas y planificar las futuras. En definitiva, todos estos procesos cognitivos hacen referencia a la habilidad que tiene el individuo de adaptarse de manera óptima a los continuos cambios del contexto para lograr superar situaciones específicas, lo que le permitirá desenvolverse con éxito en su vida diaria (Diamond, 2013; Diamond y Lee, 2016). En este sentido, y dado que la función de la Universidad es formar ciudadanos competentes que sean capaces de dar respuesta a las demandas continuamente cambiantes del contexto, desarrollar las funciones ejecutivas del alumnado se convierte en una prioridad del sistema educativo universitario. Así, un buen funcionamiento ejecutivo se asocia con un buen aprendizje autónomo, lo que a su vez contribuye de manera importante a un buen rendimiento académico y éxito laboral en la adultez, así como a un nivel socioeconómico mayor (Miyake y Friedman, 2012; Müller y Kerns, 2015).

Esta relevancia de las funciones ejecutivas queda asimismo patente en la abundante proliferación de trabajos que en los últimos años se han publicado sobre las mismas. Sin embargo, las funciones ejecutivas constituyen un área de estudio donde los aspectos conceptuales se encuentran en mejor estado de conocimiento que los aspectos referidos a su desarrollo y medición (Willoughby, Holochwost, Blanton y Blair, 2014). Así, son muy escasos los instrumentos de evaluación de las funciones ejecutivas en contextos educativos; y los existentes consisten por lo general en cuestionarios contestados por terceros informantes (frecuentemente, profesorado), por lo que su fiabilidad puede quedar en entredicho, especialmente debido a la influencia de la deseabilidad social, posibles fallos de recuerdo y memoria de los informantes e incluso falta de familiaridad y sensibilidad para percibir y detectar determinadas conductas del sujeto evaluado. Además, dichos instrumentos generalmente están compuestos por numersos items y son comercializados implicando un alto coste económico, por lo que resultan muy costosos tanto en términos temproales como económicos (más aún teniendo en cuenta la situación de crisis en la que se encuentra la universidad española actualmente).

(cc) EY-NC-ND 2018, UniversitatPolitècnica de València 
De cara a superar estas limitaciones, y en especial aquella que hace referncia a la posible fiabilidad limitada de la información recogida a través de terceros, la literatura aboga por la construcción y uso de herramientas de autoevaluación donde sea el mismo individuo quien informa de su propio funcionamiento ejecutivo en su contexto educativo, pudiendo ser ayudado para ello por el profesorado en caso de que sea necesario (Willoughby et al., 2014). Es en esta línea en la que se sitúa el presente proyecto, al tener como objetivo la traducción, adaptación y validación a población universitaria de nuevo ingreso en la Universidad de Zaragoza del "Amsterdam Executive Funtioning Inventory" (AEFI) de Baars, Bijvank, Tonnaer y Jolles (2015), destinado a la evaluación de las funciones ejecutivas, a contestar por el propio alumnado. Dicho instrumento está compuesto por 10 ítems convirtiéndolo así en una herramienta muy optimizada, además de tener acceso gratuito.

Disponer de una versión validada en castellano de esta herramienta para la evaluación de las funciones ejecutivas de nuestros estudiantes al comienzo de sus estudios, permitirá conocer las habilidades que se encuentran implicadas en su aprendizaje autónomo, lo que a su vez posibilitará detectar alumnado con probabilidades de presentar dificultades en sus estudios universitarios y bajo rendimiento académico, cuestiones que pueden llevar incluso al abandono. Sin embargo, la detección temprana de estos estudiantes a través de la herramienta a validar permitirá diseñar e implementar acciones de intervención destinadas a la mejora de sus habilidades implicadas en su aprendizaje autónomo y en última isntancia, a la mejora de su aprendizaje y rendimiento. Estas acciones podrán formar parte del Plan de Orientación Universitaria que está desarrollando la Universidad de Zaragoza, ya que mejorar el rendimiento académico de los estudiantes constituye un aspecto clave en las metas de las políticas públicas a nivel nacional e internacional y por tanto, también de nuestra universidad. En este sentido, consideramos que este proyecto puede contribuir a ello, más aún teniendo en cuenta que se dirige a estudiantes de nuevo ingreso y es entonces, durante el primer año universitario, cuando se produce el mayor desajuste alumno-contexto de aprendizaje y consecuentemente, la mayor tasa de abandono universitario (Esteban, Bernardo y Rodríguez-Muñiz, 2016). La tasa de abandono constituye un indicador de la calidad de las instituciones universitarias, conllevando además numerosos costos personales, económicos y sociales (Colás, 2015). Queda patente, por tanto, el interés de este proyecto, que supera los beneficios a corto plazo para los estudiantes y su formación, alcanzando beneficios institucionales y sociales.

\section{Objetivo}

El objetivo de este trabajo es traducir, adaptar y validar para la población universitaria de nuevo ingreso en la Universidad de Zaragoza el instrumento de evaluación de las funciones ejecutivas Amsterdam Executive Function Inventory (AEFI; Baars et al., 2015) que permita identificar alumnado con dificultades en las mismas y por tanto, alumnado con probabilidad de presentar problemas en su aprendizaje autónomo. Se trata de un instrumento compuesto por tres dimensiones y 10 ítems: atención (ítems 3, 6 y 9), autocontrol (ítems 1, 5, 8 y 10) y planificación (ítems 2, 4 y 7). Las respuestas a los ítems del AEFI se recogen en una escala

(cc) BY-NC-ND 2018, UniversitatPolitècnica de València

Congreso IN-RED (2018) 
tipo Likert de 3 puntos con las siguientes opciones de elección: 1 = "no verdadero", 2 = "parcialmente verdadero", y 3 = "verdadero".

\section{Desarrollo de la innovación}

Para llevar a cabo esta experiencia se ha seguido el procedimiento que se indica a continuación, en el cual puede distinguirse dos grandes fases destinadas a la: 1) traducción y adaptación del instrumento y 2) validación del instrumento.

1.- Traducción y adaptación del instrumento original.

Para llevar a cabo la traducción del cuestionario AEFI se siguió el método de traducción directa/inversa recomendado en la literatura científica especializada (Forsyth y Lessler, 1991; Hambleton, 2005; Hambleton y Patsula, 1999). Para ello, dos traductores independientes tradujeron individualmente la versión original del AEFI al castellano, originándose así las denominadas versión 1 y versión 2 del intrumento en castellano. Cada una de estas dos versiones en castellano fueron evaluadas a través del juicio de tres expertos (profesorado universitario con una experiencia media de 24,6 años en Psicología de la Educación, aprendizaje y funciones ejecutivas; oscilando su experiencia entre 14 y 35 años), resultando la versión 3 en castellano del AEFI que fue utilizada para determinar la validez de contenido. Para ello, participó un nuevo grupo formado por siete expertos, todos profesores universitarios con una experiencia media en el ámbito de la Educación Superior de 20,5 años (oscilando su experiencia entre 8,5 años y 30 años). Cada uno de estos siete expertos valoró ésta versión 3 del instrumento cumplimentando una plantilla relativa a la claridad de cada ítem entendiédose que un ítem presenta claridad cuando este se comprende fácilmente, es decir, su sintáctica y semántica son adecuadas. Así, se utilizó la siguiente escala según la propuesta de Escobar y Cuervo (2008): 1 No cumple con el criterio: el ítem no es claro; 2 Bajo nivel: el ítem requiere bastantes modificaciones o una modificación muy grande en el uso de las palabras de acuerdo con su significado o por la ordenación de las mismas; 3 Moderado nivel: se requiere una modificación muy específica de algunos de los términos del ítem; y 4 Alto nivel: el ítem es claro, tiene semántica y sintaxis adecuada. Todos los items resultaron ser claros, obteniéndose una puntuación media de 3,56 y desviación típica 0,33.

A partir de esta versión 3 del instrumento en castellano, otros dos profesores universitarios y bilingües llevaron a cabo una retrotraducción de manera independiente. Es decir, cada uno de ellos realizó una retrotraducción de la versión 3 en castellano al inglés, originándose así las versiones 1 y 2 del instrumento en inglés. Posteriormente, estas mismas personas pusieron en común sus versiones y discutieron sobre las mismas hasta llegar a una versión consensuada: versión 3 del instrumento en inglés.

Posteriormente, una persona bilingüe y experta en Psicología de la Educación, comparó la versión original del instrumento AEFI con la versión 3 del instrumento en inglés, considerándolas equivalentes. Por tanto, se obtuvo así la versión 4 del instrumento en castellano a administrar al alumnado para proceder posteriormente a su validación.

(cc) EY-NC-ND 2018, UniversitatPolitècnica de València 
Los ítems empleados para la recogida de datos tras el proceso de traducción y adaptación fueron los siguientes:

1. A menudo reacciono demasiado rápido. Hago o digo algo antes de que sea mi turno.

2. Me organizo bien. Por ejemplo, planifico adecuadamente lo que realizaré a lo largo del día.

3. No soy capaz de concentrarme en el mismo tema durante un largo periodo de tiempo.

4. Trabajo de manera muy ordenada.

5. Comparado con otros, hablo mucho.

6. Me distraigo fácilmente.

7. Soy caótico o desorganizado.

8. Antes de actuar no pienso en las consecuencias de mis actos.

9. Mi pensamiento se dispersa fácilmente.

10. Soy un bocazas, hablo más de la cuenta y de forma indiscreta.

2.- Validación del cuestionario.

La versión 4 del instrumento en castellano fue adminsitrada a una muestra de 148 estudiantes de nuevo ingreso en la Universidad de Zaragoza, pertenecientes al Grado en Magistero en Educación Infantil. Todos eran de género femenino salvo uno y respondieron de forma voluntaria y anónima al cuestionario, que fue administrado en los últimos 10 minutos de una clase habitual en el mes de diciembre de 2017. Los estudiantes pertenecían a los dos grupos del Grado existentes en el centro.

Se efectuaron análisis descriptivos de los ítems. Se calculó la pertinencia del análisis factorial exploratorio (AFE) y se finalizó con un análisis factorial confirmatorio (AFC) de la escala mediante el software AMOS 20.0. La bondad de ajuste del modelo de medida propuesto se evaluó mediante diferentes pruebas, utilizando para la estimación de parámetros la técnica de máxima verosimilitud. Por un lado, los índices de ajuste global o absoluto fueron el valor de chi-cuadrado $\left(\chi^{2}\right)$, la ratio $\chi^{2} / g l$, y los índices RMSR (Root Mean Square Residual) y RMSEA (Root Mean Square Error or Approximation), mientras que los índices de ajuste incremental o de comparación empleados fueron TLI (Tucker-Lewis Index), IFI (Incremental Fit Index) y CFI (Comparative Fit Index). Para la ratio $\chi^{2} / \mathrm{gl}$, un valor inferior a 3 supone un indicativo de un buen ajuste (Kline, 2005). Los valores de los índices TLI, IFI y CFI deben ser superiores a .90 para considerar aceptable el ajuste del modelo (Hair, Black, Babin, Anderson y Tatham, 2006). El índice RMSEA es considerado el mejor indicador del ajuste global (Marsh, Balla y Hau, 1996) y al igual que el índice RMSR, valores entre .05 y .08 son considerados como aceptables, mientras que valores inferiores a .05 se consideran óptimos (Batista y Coenders, 2012).

(cc) EY-NC-ND 2018, UniversitatPolitècnica de València

Congreso IN-RED (2018) 


\section{Resultados}

Los estadísticos descriptivos de los ítems mostraron valores de asimetría y curtosis entre 3 y 10 respectivamente, cumpliendo así el rango de normalidad (Marôco, 2010). Los valores medios más elevados se corresponden con dos ítems pertenecientes a la dimensión Planificación (ítem 2: 2,43; ítem 4: 2,49). El resto de ítems obtuvieron una valoración por encima del punto medio de la escala. La pertinencia del AFE mostró un índice de adecuación muestral $\mathrm{KMO}=.72 \mathrm{y}$ significatividad en el test de Esfericidad de Bartlett $\left[\chi^{2}(45)=289.34 ; p<.01\right]$. Utilizando una rotación oblícua Oblimin, se obtuvo una solución de tres factores que explicaban el $57,7 \%$ de la varianza. El análisis de fiabilidad mostró un valor $\alpha$ de .71 para la escala global.

El AFC realizado para el modelo de medida indicó un ajuste a los datos satisfactorio, situándose los diferentes índices considerados en el análisis dentro del rango aceptable $\chi^{2}(32)=47.01(\mathrm{p}<.001) ; \chi^{2} / \mathrm{gl}=1.46 ; \mathrm{RMSR}=.026$; RMSEA=.058; TLI=.917; IFI=.944; CFI=.941]. La ratio $\chi^{2} / \mathrm{gl}$ asumió un valor inferior a 3, los valores para TLI, IFI y CFI se situarion por encima del criterio .90 recomendado, el índice RMSR obtuvo un ajuste óptimo mientras que el ajuste del índice RMSEA fue aceptable.

\section{Conclusiones}

Disponer de este instrumento de evaluación válido en nuestro contexto permitirá detectar a todos aquellos alumnos universitarios de nuevo ingreso que presentan dificultades en su aprendizaje autónomo. Además, las características particulares del instrumento (breve y gratuito) permitirán llevar a cabo esta detección de manera rápida y viable económicamente. Estas cuestiones son importantes, preocupándonos como docentes de modo especialmente lo referido al tiempo. Son muchos los estudiantes que comienzan la universidad con dificultades en su aprendizaje autónomo, con las consecuencias negativas que ello implica para su formación, rendimiento y éxito académico. Por ello, cuanto antes se lleve a cabo la detección de estos estudiantes, antes podrá intervenirse y mayores y más perdurables serán sus beneficios. Estas intervenciones podrán realizarse dentro del Plan de Orientación de la Universidad de Zaragoza (Allueva, Zulaica y Abadía, 2016), pues si bien dispone de una estructura y contenidos generales para todos los estudiantes, es altamente flexible para que cada centro lo personalice atendiendo a las necesidades y demandas de sus estudiantes.

Por otro lado, el hecho de disponer de esta herramienta válida en nuestro contexto abre interesantes y necesarias líneas de trabajo a desarrollar en futuros proyectos con la finalidad, por ejemplo, de conocer si determinadas variables del alumnado (género, edad, tipo de centro al que acudieron en niveles educativos previos, rendimiento académico previo y Grado que estudian) se asocian con una mayor probabilidad de dificultades en las funciones ejecutivas y en el aprendizaje autónomo, pudiendo establecerse un perfil de alumnado con mayor riesgo de déficits en su aprendizaje. Ello permitiría trabajar con este alumnado con potecial riesgo de presentar dificultades en su aprendizaje incluso antes de comenzar sus estudios universitarios, por ejemplo, en los denominados "Cursos Cero" que 
imparte la Universidad de Zaragoza (actividades que se realizan en el mes de septiembre y que se dirigen al alumnado de nuevo ingreso con el fin de facilitar a los estudiantes algunas herramientas para poder desarrollar con éxito sus estudios en los siguientes años).

Asimismo, el cuestionario obtenido podría validarse para estudiantes de otros cursos de la Universidad de Zaragoza, lo que permitirá hacer un seguimiento de la mejora de las funciones ejecutivas y aprendizaje autónomo de los estudiantes. También esta validación realizada con estudiantes de la Universidad de Zaragoza puede constituir el primer paso para una futura validación a nivel nacional con participación de otras Universidades.

\section{Referencias}

ALlUEVA, P., ZULAICA, F., ABADÍA, A. R. (2016). "Plan de Orientación Universitaria de la Universidad de Zaragoza. Integración de los estudiantes en la Universidad: Binomio Tutor-Mentor” en Castejón, J.L. Psicología y Educación: Presente y Futuro. Madrid: Asociación Científica de Psicología y Educación; pp. 2273-2281.

BAARS, M. A. E., BIJVANK, M. N., TONNAER, G. H., JOLLES, J. (2015). “Self-report measures of executive functioning are a determinant of academic performance in first-year students at a university of applied sciences” en Frontiers in Psychology, Vol. 6, núm. 1131. $<$ https://www.frontiersin.org/articles/10.3389/fpsyg.2015.01131/full>. [Consulta: 10 de mayo de 2017]

BATISTA, J. M., COENDERS, G. (2012). Modelos de ecuaciones estructurales ( $2^{a}$ Ed.). Madrid : La Muralla.

CARLSON, S. M., ZELAZO, P. D., FAJA, S. (2013). “Executive function” en Zelazo, P.D. The Oxford handbook of developmental psychology, Vol. 1: Body and mind. New York: Oxford University Press. (pp. 706-743).

COLÁS, P. (2015). “El abandono universitario” en Revista Fuentes, Vol. 16, pp. 9-14.

DIAMOND, A. (2013). “Executive Functions” en Annual Review of Psychology, Vol. 64, pp. 135168. <10.1146/annurev-psych-113011-143750>. [Consulta: 15 de enero de 2018]

DIAMOND, A., LEE, D. S. (2016). “Conclusions about interventions, programs, and approaches for improving executive functions that appear justified and those that, despite much hype, do not” en Developmental Cognitive Neuroscience, Vol. 18, pp. 34-48.

ESCOBAR, J., CUERVO, A. (2008). "Validez de contenido y juicio de expertos: una aproximación a su utilización” en Avances en Medición, Vol. 6, núm. 1, pp. 27-36. <http://www.humanas.unal.edu.co/psicometria/files/7113/8574/5708/Articulo3_Juicio_de_expertos_2 7-36.pdf> [Consulta: 12 de enero de 2018]

ESTEBAN, M., BERNARDO, A. B., RODRÍGUEZ-MUÑIZ, L. J. (2016). "Permanencia en la universidad: la importancia de un buen comienzo”en Aula Abierta, Vol. 44, pp. 1-6.

FORSYTH, B. H., LESSLER, J. T. (1991). “Cognitive laboratory methods: a taxonomy” en Bierner, P. et al. Measurement errors in surveys. NewYork: Wiley; pp. 393-418.

FRANCIS, A., FLANIGAN, A. (2012). "Self-Directed Learning and Higher Education Practices: Implications for Student Performance and Engagement” en International Journal of the Scholarship of Teaching and Learning, Vol. 7, núm. 3, pp. 1-18.

(cc) EY-NC-ND 2018, UniversitatPolitècnica de València

Congreso IN-RED (2018) 
HAIR, J. F., BLACK, W. C., BABIN, B., ANDERSON, R. E., TATHAM, R. L. (2006). Multivariate Data Analysis (6 ${ }^{a}$ Ed.). Upper Saddle River, NJ: Prentice-Hall.

HAMBLETON, R. K. (2005). "Issues, designs, and technical guidelines for adapting tests into multiple languages and cultures” en Hambleton, R.K., Merenda, P., Spielberger, C. Adapting educational and psychological tests for cross-cultural assessment. Mahwah, NJ: Lawrence Erlbaum; pp. 3-38.

HAMBLETON, R. K., PATSULA, L. (1999). “Increasing the Validity of Adapted Tests: Myths to be Avoided and Guidelines for Improving Test Adaptation Practices” en Journal of Applied Testing Technology, $\quad$ Vol. $\quad 1, \quad$ núm. $\quad 1, \quad$ pp. 1-13. $<$ http://www.jattjournal.com/index.php/atp/article/view/48345/39215>. [Consulta: 20 de mayo de 2017]

KLINE, R. B. (2005). Principles and practice of structural equation modeling ( ${ }^{a}$ Ed.). New York: The Guilford Press.

MARÔCO, J. (2010). Análise de Equações Estructurais. Fundamentos teóricos, Software \& Aplicações. Pero Pinheiro: Rolo y Filhos II, SA.

MARSH, H., BALLA, J. R., HAU, K. T. (1996). "An evaluation of incremental fit indices: A clarification of mathematical and empirical processes” en Marcoulides, G.A., Schumacker, R.E. Advanced structural equation modeling techniques. Hilsdale, NJ: Erlbaum; pp. 115-353.

MIYAKE, A., FRIEDMAN, N. P. (2012). "The nature and organization of individual differences in executive functions: four general conclusions” en Current Directions in Psychological Science, Vol. 21, pp. 8-14. <10.1177/0963721411429458>. [Consulta: 15 de enero de 2018]

MÜLLER, U., KERNS, K. A. (2015). “The development of executive function” en Liben, L.S., Müller, U., Lerner, R.M. Handbook of Child Psychology and Developmental Science: Cognitive Processes. Hoboken, NJ: John Wiley and Sons; pp. 571-623.

POZO, J. I., MONEREO, J. I. (2009). “Introducción: La nueva cultura del aprendizaje universitario o por qué cambiar nuestras formas de enseñar y aprender” en Pozo, J.I., Pérez Echeverría, M.P. Psicología del aprendizaje universitario: La formación en competencias. Madrid: Morata; pp. 9-28.

RUÉ, J. (2009). El aprendizaje autónomo en educación superior. Madrid: Narcea.

WILLOUGHBY, M. T., HOLOCHWOST, S. J., BLANTON, Z. E., BLAIR, C. B. (2014). "Executive Functions: Formative versus Reflective measurement” en Measurement: Interdisciplinary Research and Perspectives, Vol. 12, núm. 3, pp. 69-95. <10.1080/15366367.2014.929.453>. [Consulta: 10 de diciembre de 2017]

ZELAZO, P. D., BLAIR, C. B., WILLOUGHBY, M. T. (2016). Executive Function: Implications for Education (NCER 2017-2000). Washington, DC: National Center for Education Research, Institute of Education Sciences, U.S. Department of Education. <http://ies.ed.gov/> [Consulta: 15 de enero de 2018] 